\title{
DESIGN OF A NOVEL BIOACTIVE CALCIUM PHOSPHATE PASTE CONTAINING ACETYL CELLULOSE
}

\author{
Takahiro Kawai, ${ }^{1 *}$ Chikara Ohtsuki, ${ }^{1}$ Hirofumi Inada, ${ }^{1}$ Masanobu Kamitakahara, ${ }^{1}$ \\ Masao Tanihara, ${ }^{1}$ Toshiki Miyazaki ${ }^{2}$ \\ (*Corresponding author: kaw-taka@,ms.naist.jp) \\ ${ }^{1}$ Graduate School of Materials Science, Nara Institute of Science and Technology \\ 8916-5 Takayama, Ikoma, Nara 630-0192, Japan \\ ${ }^{2}$ Graduate School of Life Science and Systems Engineering, Kyushu Institute of Technology \\ 2-4 Hibikino, Wakamatsu, Kitakyushu, Fukuoka 808-0196, Japan
}

Keywords: Calcium phosphate paste, Acetyl cellulose, Hydroxyapatite, Mechanical properties, Simulated body fluid

\begin{abstract}
A novel self-curing paste, which is composed of calcium phosphates powder and acetyl cellulose dissolved in ethyl lactate, has been developed as a bone-repairing material that does not need any mixing operation before its use and sets in the body. This paper focused on investigations of the mechanical properties and bioactivity of the paste after exposure to physiological solutions. Compressive strength of the set paste increased with increment of the polymer concentration and of calcium phosphates powder/polymer solution $(\mathrm{P} / \mathrm{L})$ ratio, and strain at the yield point increased with increasing the polymer concentration and with decreasing $\mathrm{P} / \mathrm{L}$. The mechanical properties of the set paste including calcium phosphates and acetyl cellulose were analogous to those of human cancellous bone. The paste was able to form bone-like hydroxyapatite on its surface in a simulated body fluid (SBF). This indicates that the paste has a potential to show bone-bonding ability, i.e. bioactivity. From these results, this paste can achieve both the mechanical properties corresponding to human cancellous bone and show bioactivity as well as easy handling.
\end{abstract}

(Received June 30, 2004; Accepted August 25, 2004)

\section{INTRODUCTION}

Bioactive self-curing pastes are attractive materials since they can be injected into a bony defect and achieve a complete packing of the bony defect even where its shape is complex. Many studies are reported on bioactive self-curing pastes that can be easily shaped and set in a living body after the implantation. A paste consisting of powder mixture of dicalcium phosphate anhydrous (DCPA) and tetracalcium phosphate (TTCP), and water is a typical example of bioactive self-curing fillers for bone-repairing. ${ }^{1-4}$ This paste sets through a reaction of the conversion of DCPA and TTCP into hydroxyapatite. Such a type of calcium phosphate paste is widely used in clinical applications. ${ }^{5,6}$ There still remain difficulties on the use of the conventional calcium phosphate paste that a powder component has to be mixed with a liquid component homogeneously within a short period in order to achieve high performances including easy handling and high mechanical strength. Therefore, a novel bioactive paste that does not require the mixing procedure during operations is desired. In the present study, we propose a novel bioactive paste by mixing DCPA and TTCP with acetyl cellulose dissolved in ethyl lactate. When this bioactive paste is exposed to body fluid, ethyl lactate in the paste is gradually replaced by water and acetyl cellulose precipitates in the paste since acetyl cellulose does not dissolve in water. At the same time, DCPA and TTCP were reacted with water to set by formation of hydroxyapatite. The set paste is expected to show higher toughness than conventional calcium phosphate paste, because it is a composite of inorganic and organic components. This study focused on fabrication of such a type of paste, and on evaluation of its mechanical properties and apatite-forming ability in physiological solutions.

\section{EXPERIMENTAL PROCEDURE}

\section{Preparation of calcium phosphates powder}

DCPA powder was obtained by firing dicalcium hydrogenphosphate dihydrate (DCPD; Nacalai Tesque, Inc., Japan) at $250^{\circ} \mathrm{C}$ for $10 \mathrm{~h}$ (heating rate; $5^{\circ} \mathrm{C} / \mathrm{min}$ ) in a muffle furnace (KDF S-70G, Denken Co., Ltd., Japan). TTCP, powder was prepared by heating an equimolar mixture of DCPA and calcium carbonate $\left(\mathrm{CaCO}_{3}\right.$; Nacalai Tesque, Inc., Japan) at $1500^{\circ} \mathrm{C}$ for $24 \mathrm{~h}$ (heating rate; $5^{\circ} \mathrm{C} / \mathrm{min}$ ) in a $\mathrm{MoSi}_{2}$ furnace (SB-2025D, Motoyama Co., Ltd., Japan), followed by rapid cooling to the room temperature, according to a previous literature. ${ }^{7}$ After grinding the TTCP with a mortar (Type AGA, Ishikawa Kojo Co., Ltd., Japan) for 30 minutes, the obtained TTCP powder was sieved to less than 150 $\mu \mathrm{m}$. DCPA powder was also sieved to obtain particles 
Kawai T, Ohtsuki C, Inada H, Kamitakahara M, Tanihara M, Miyazaki T

with their sizes of less than $45 \mu \mathrm{m}$. Equimolar mixture of DCPA and TTCP (denoted as calcium phosphates powder) was then prepared with a mortar.

\section{Preparation of polymer solution}

10,30 or $50 \mathrm{~g}$ of acetyl cellulose (Average molecular weight; ca. 40,000, Wako pure chemicals, Co., Ltd., Japan) was dissolved in $100 \mathrm{~mL}$ of ethyl lactate (Wako pure chemicals, Co., Ltd., Japan) at $60^{\circ} \mathrm{C}$. The obtained solutions are denoted as CA10, CA30 and CA50, respectively, and ethyl lactate solution without acetyl cellulose as CA0.

\section{Preparation of paste}

The calcium phosphates powder was mixed with the polymer solution with different concentrations, where the mass ratio of powder to liquid $(\mathrm{P} / \mathrm{L})$ was 2. The obtained paste is denoted as CP2CAy $(y=0$, $10,30$ and 50$)$. The calcium phosphates powder was also mixed with CA50 with $\mathrm{P} / \mathrm{L}=0$ and 1 . They are denoted as CPxCA50 ( $x=0$ and 1). The powder and solution were manually mixed for $10 \mathrm{~min}$.

4. Soaking in a buffered solution and a simulated body fluid

The paste was packed into a silicone tube whose diameter and height were $6 \mathrm{~mm}$ and $12 \mathrm{~mm}$, respectively, and soaked in $40 \mathrm{~mL}$ of Tris- $\mathrm{NaCl}$ buffer solution (TRS; $\mathrm{Na}^{+} 142.0$ and $\mathrm{Cl}^{-} 142.0$ $\mathrm{mol} \cdot \mathrm{m}^{-3}$ ), or a simulated body fluid (SBF; $\mathrm{Na}^{+} 142.0$, $\mathrm{K}^{+}$5.0, $\mathrm{Mg}^{2+} 1.5, \mathrm{Ca}^{2+} 2.5, \mathrm{Cl}^{-} 147.8, \mathrm{HCO}_{3}^{-} 4.2$, $\mathrm{HPO}_{4}{ }^{2-} 1.0$, and $\mathrm{SO}_{4}{ }^{2-} 0.5 \mathrm{~mol} \cdot \mathrm{m}^{-3}$ ) whose ion concentrations are nearly equal to those of human blood plasma at $36.5^{\circ} \mathrm{C}$. TRS was prepared by dissolving reagent grade chemicals of $\mathrm{NaCl}$ in ultrapure water, and $\mathrm{SBF}$ by $\mathrm{NaCl}, \mathrm{NaHCO}_{3}, \mathrm{KCl}$, $\mathrm{K}_{2} \mathrm{HPO}_{4} \cdot 3 \mathrm{H}_{2} \mathrm{O}, \mathrm{MgCl}_{2} \cdot 6 \mathrm{H}_{2} \mathrm{O}, \mathrm{CaCl}_{2}$ and $\mathrm{Na}_{2} \mathrm{SO}_{4}$. The $\mathrm{pH}$ of these solutions were buffered at 7.25 using $50 \mathrm{~mol} \cdot \mathrm{m}^{-3}$ of tris (hydroxymethyl) aminomethane along with appropriate volume of 1 $\mathrm{kmol} \cdot \mathrm{m}^{-3}$ hydrochloric acid solution. The temperature of the solutions were kept at $36.5^{\circ} \mathrm{C} .^{8,9}$

\section{Measurement of mechanical properties}

After soaking in the TRS for 7 days, the specimen was taken out from the solution to measure compressive strength. The measurements were performed using Universal Testing Machine (Type5566, Instron Japan Co., Ltd., Japan) just after the specimen was taken out from the solution. The compressive load was applied to each specimen at a cross-head speed of $20 \mathrm{~mm} / \mathrm{min}$. The compressive strength and Young's modulus were defined as the value of stress at the first yield point and as the slope of the linear portion at the initial stage of the obtained stress-strain curve, respectively.

\section{Evaluation of structure of set pastes}

The structures of the set pastes after soaking in TRS were analyzed by powder XRD (MXP3V, MAC Science Co., Ltd., Japan) after grinding with a mortar. The surfaces and cross-sections of the set pastes after soaking in TRS or SBF were observed by scanning electron microscopy (SEM; S-3500N, Hitachi, Ltd., Japan).

\section{RESULTS}

\section{Mechanical properties of set pastes}

The specimen CP2CA0 was difficult to be packed in a silicone tube due to its high mobility, and its assessment was impossible. Figure 1 shows representative stress-strain curves of the set paste CP2CAy after soaking in TRS for 7 days. The shape of each of the curves looks similar but the compressive strength and strain at the yield point increased with increasing the polymer concentration. These three pastes with different polymer concentrations showed almost the same Young's modulus. Figure 2 shows representative stress-strain curves of the set paste CPxCA50 after soaking in TRS for 7 days. The compressive strength and Young's modulus increased with increasing $\mathrm{P} / \mathrm{L}$ ratio, and the strain at the yield point decreased with increasing $\mathrm{P} / \mathrm{L}$ ratio. The data of their mechanical properties, in comparison with human cancellous bone, are summarized in Table 1. These results indicate compressive strength and strain at the yield point were determined depending on both polymer concentration and $\mathrm{P} / \mathrm{L}$ ratio, but Young's modulus on $\mathrm{P} / \mathrm{L}$ ratio only.

\section{Structure of set pastes}

Figure 3 shows SEM images of the surfaces of the pastes after soaking in TRS for 7 days. Particles with their sizes between 10 and $20 \mu \mathrm{m}$ were observed to aggregate for every specimen, except for CP0CA50 whose surface looks completely smooth. The grain boundaries on the surface look more distinct with increasing the polymer concentration or $\mathrm{P} / \mathrm{L}$ in the paste. Figure 4 shows SEM images of the cross-section of CP2CA50 after soaking in TRS for 7 days. The grain boundaries look much clearer than those on the surface. From the image with higher magnification for the cross-section, needle-like depositions were observed on the grains in some parts. Figure 5 shows SEM images of the surfaces of CP2CAy and CPxCA50 after soaking in SBF for 7 days. Plate-like particles were observed to cover all areas on the surfaces for every specimen, except for CP0CA50. On the surface of CP0CA50, no precipitate was observed. Morphology of the 


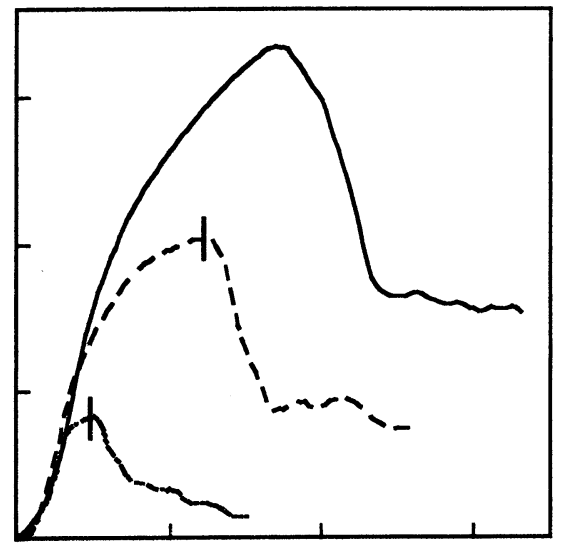

Fig. 1 Stress-strain curves of CP2CAy $(y=10,30$ and 50) after soaking in TRS for 7 days. Lines drawn vertically to the horizontal axis show their first yield points.

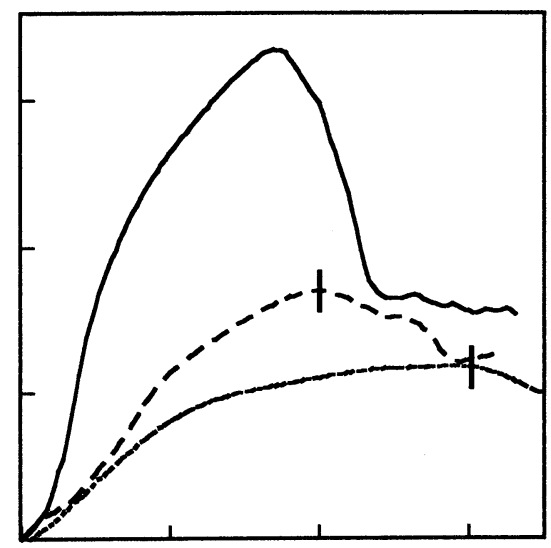

Fig. 2 Stress-strain curves of CP $x$ CA50 ( $x=0,1$ and 2) after soaking in TRS for 7 days. Lines drawn vertically to the horizontal axis show their first yield points.

Table 1 Mechanical properties of the specimens after soaking in TRS for 7 days, in comparison with those of human cancellous bone

\begin{tabular}{cccc}
\hline Specimen & $\begin{array}{c}\text { Compressive strength } \\
/ \mathrm{MPa}\end{array}$ & $\begin{array}{c}\text { Strain at the yield } \\
\text { point } / \%\end{array}$ & $\begin{array}{c}\text { Young's modulus } \\
/ \mathrm{MPa}\end{array}$ \\
\hline CP2CA10 & $3.9 \pm 0.4$ & $3.9 \pm 2.1$ & $130.9 \pm 16.2$ \\
CP2CA30 & $10.2 \pm 1.9$ & $8.0 \pm 2.3$ & $143.1 \pm 12.2$ \\
CP2CA50 & $16.9 \pm 0.8$ & $15.7 \pm 1.3$ & $140.5 \pm 15.9$ \\
CP1CA50 & $8.7 \pm 0.3$ & $19.8 \pm 2.8$ & $57.1 \pm 5.6$ \\
CP0CA50 & $7.1 \pm 1.3$ & $29.4 \pm 5.4$ & $37.3 \pm 4.0$ \\
Human cancellous bone & $2-12$ & $5-7$ & $50-500$ \\
\hline
\end{tabular}

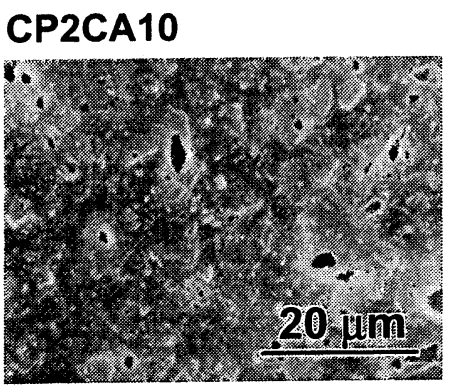

CP1CA50

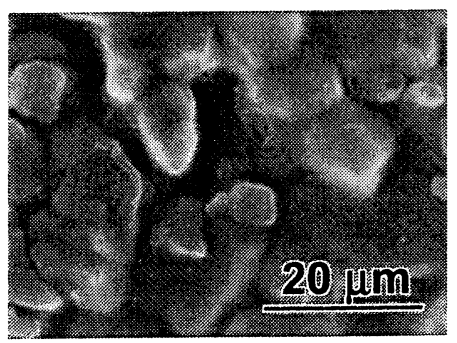

CP2CA50

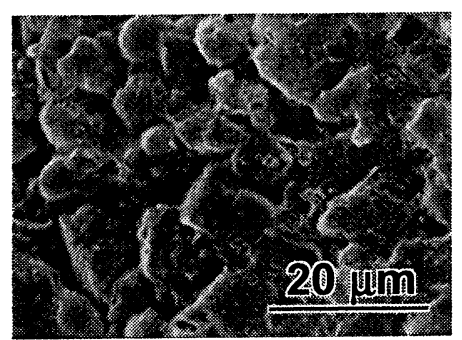

CPOCA50

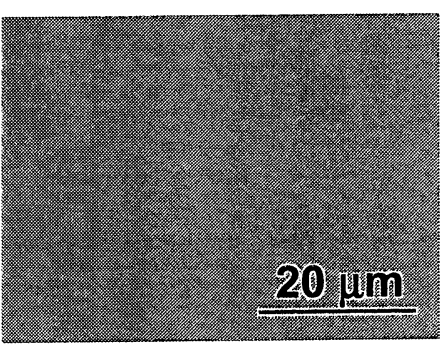

Fig. 3 SEM images of the surfaces of the pastes after soaking in TRS for 7 days.

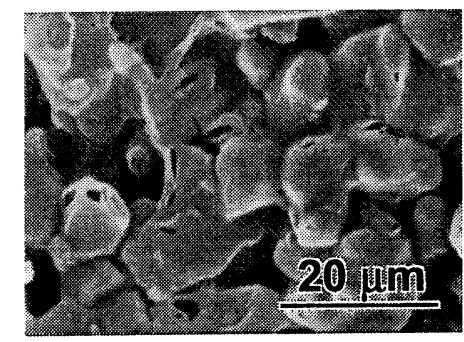



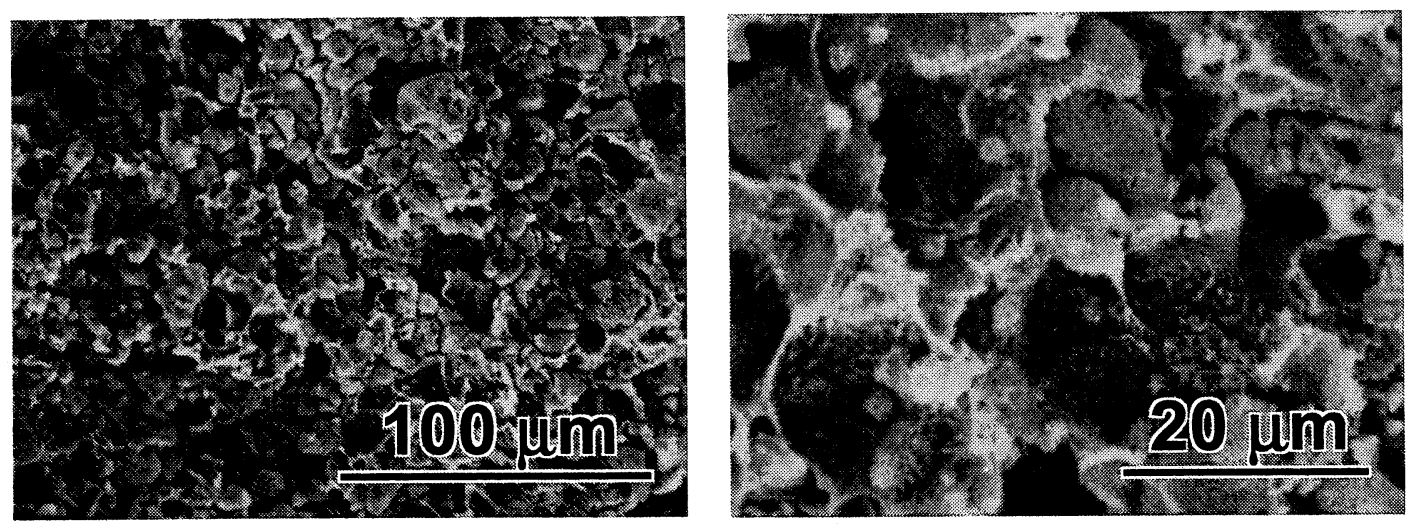

Fig. 4 SEM images of the cross-section of CP2CA50 after soaking in TRS for 7 days (Left; low, Right; high magnification).

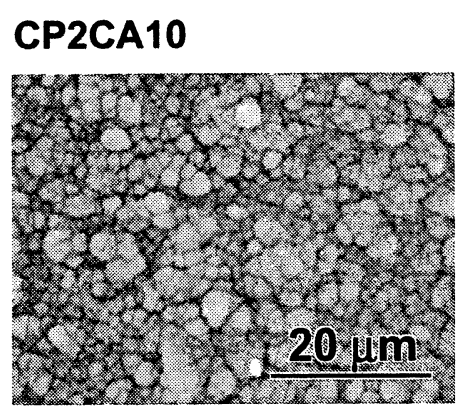

CP1CA50

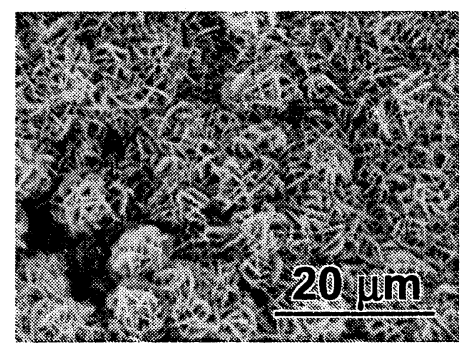

CP2CA30

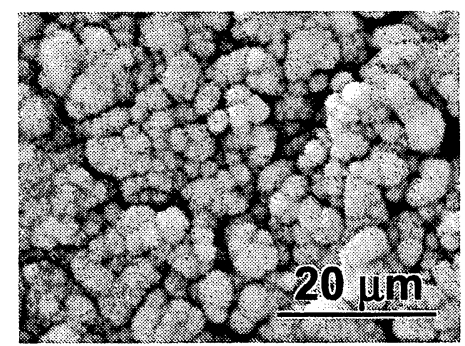

\section{CP2CA50}

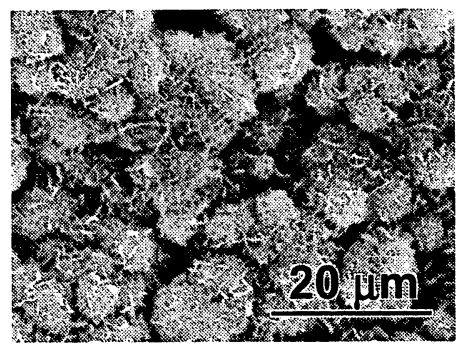

\section{CP0CA50}

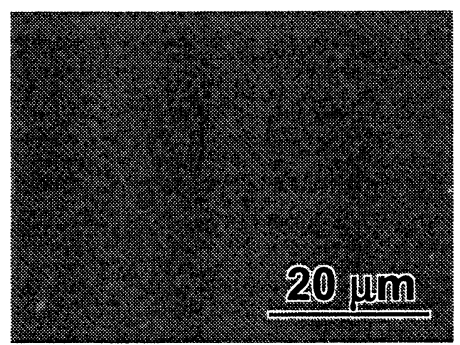

Fig. 5 SEM images of the surfaces of the pastes after soaking in SBF for 7 days.

deposited particles is similar to that of bone-like apatite formed on the bioactive materials in SBF. ${ }^{8}$

Figure 6 shows powder XRD patterns of CP2CAy after soaking in TRS for 7 days. Peaks assigned to hydroxyapatite were detected for each diffraction pattern after soaking in TRS, while the intensities of the TTCP and DCPA peaks decreased in comparison with those of calcium phosphates powder before soaking. This indicates that TTCP and DCPA in the pastes were gradually reacted to convert to hydroxyapatite in TRS. The broadness of the peaks between $31^{\circ}$ and $33^{\circ}$ can be seen for each pattern, indicating that hydroxyapatite precipitated in the set paste has low crystallinity. In the case of
CP2CA50, the peak intensities of hydroxyapatite were lower than those of CP2CA10 or CP2CA30.

\section{DISCUSSION}

Even CP0CA50, free from calcium phosphates, set in TRS or SBF, indicating that acetyl cellulose precipitated by the replacement of ethyl lactate by water. Acetyl cellulose precipitates as it fills gaps between the particles of TTCP and DCPA. At the same time, calcium phosphates powder reacts to set by producing hydroxyapatite with water. A number of studies concerning calcium phosphates paste have reported $^{1-3,10,11}$ the reaction between TTCP and DCPA in 


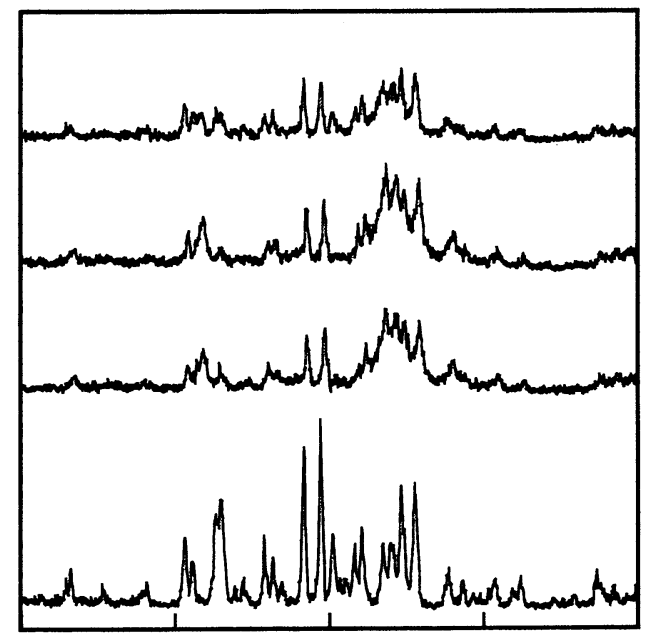

Fig. 6 Powder XRD patterns of CP2CAy $(y=10,30$ and 50) after soaking in TRS for 7 days. CP powder indicates powder mixture of DCPA and TTCP without exposure to TRS.

water to transform to hydroxyapatite shown as the formula below.

$$
2 \mathrm{Ca}_{4} \mathrm{O}\left(\mathrm{PO}_{4}\right)_{2}+2 \mathrm{CaHPO}_{4} \rightarrow \mathrm{Ca}_{10}\left(\mathrm{PO}_{4}\right)_{6}(\mathrm{OH})_{2}
$$

Results of XRD clearly indicate the present paste set through the reaction. SEM observation of the cross-section shows the presence of needle-like particles among the grains in the paste. Morphology of these deposits is quite similar to the hydroxyapatite formed in the paste prepared only with calcium phosphates powder after exposure to physiological conditions. ${ }^{12,13}$. In the case of P/L of 2, unreacted TTCP and DCPA were detected by the XRD for the present pastes even after soaking TRS for 7 days, as seen on Fig. 6, and this suggests that a reaction did not proceed well inside of this specimen. This should be due to covering of the surfaces of calcium phosphates powder with the precipitated acetyl cellulose, as shown in Fig. 3. Therefore the conversion to hydroxyapatite progressed for the pastes with comparatively lower concentrations of polymer such as CP2CA10 and CA2CA30.

Compressive strength increased with increasing polymer concentration in the paste. The higher concentration of polymer was used, the denser material after setting was produced. In the case of $\mathrm{P} / \mathrm{L}$ of 2 , all the specimens showed similar Young's modulus regardless of the polymer concentration. When the higher calcium phosphates powder content applied, the powders well packed to contact with each other independently on polymer concentration, and the stress concentrates on the powders at the loading. In contrast, when the specimen has smaller $\mathrm{P} / \mathrm{L}$, the distances between inorganic powders become larger. In this case, the stress concentrates mainly on polymer region, which forms the continuous matrix, and hence Young's modulus decreases with decreasing $\mathrm{P} / \mathrm{L}$. The compressive strength and Young's modulus of the specimens CP2CA30, CP2CA50 and CP1CA50 after soaking in TRS for 7 days reached to those of human cancellous bone, and strain at the yield point was larger than that of human cancellous bone. ${ }^{14}$

After the soaking in SBF, the specimens with both calcium phosphates powder and acetyl cellulose deposited bone-like apatite on their surfaces, suggesting they have a potential to show bone-bonding ability, i.e. bioactivity. The hydroxyapatite, which was formed on their surfaces by the reaction of DCPA and TTCP, was suggested to grow by consuming calcium and phosphate ions in SBF, on the basis of similar mechanism reported previously. ${ }^{8}$

\section{CONCLUSION}

The paste consisting of calcium phosphates powder and acetyl cellulose dissolved in ethyl lactate set in a physiological solution. The paste fabricated in this study is expected as a promising material showing mechanical properties analogous to those of human cancellous bone and having a potential to show 
Kawai T, Ohtsuki C, Inada H, Kamitakahara M, Tanihara M, Miyazaki T

bioactivity as well as easy handling.

\section{REFERENCES}

1. C. Liu, W. Shen, Y. Gu, L. Hu, J. Biomed. Mater. Res., 35, 75 (1997).

2. K. Ishikawa, S. Takagi, L.C. Chow, K. Suzuki, J. Biomed. Mater. Res., 46, 504 (1999).

3. S. Takagi, L.C. Chow, K. Ishikawa, Biomaterials, 19, 1593 (1998).

4. L.C. Chow, S. Hirayama, S. Takagi, E. Parry, J. Biomed. Mater. Res., 53, 511 (1998).

5. J.F. Kveton, C.D. Friedman, P.D. Costantino, Am. J. Otl., 16, 465 (1995).

6. C.D. Friedman, P.D. Costantino, C.H. Snyderman, L.C. Chow, S. Takagi, Arch. Facial. Plast. Surg., 58, 124 (2000).

7. W.E. Brown, E.F. Epstein, J. Res. Natl. Bur. Stand., 69A, 547 (1965).

8. M. Tanahashi, T. Yao, T. Kokubo, M. Minoda, T. Miyamoto, T. Nakamura, T. Yamamuro, J. Am. Ceram. Soc., 77, 2805 (1994).

9. C. Ohtsuki, T. Kokubo, M. Neo, S. Kotani, T. Yamamuro, T. Nakamura, Y. Bando, Phosphorus Res. Bull., 1, 191 (1991).

10. U. Gbureck, J. Probst, R. Thull, Biomol. Eng., 19, 51 (2002).

11. C. Liu, H. Shao, F. Chen, H. Zheng, Biomaterials, 24, 4103 (2004).

12. S. Takagi, L.C. Chow, M. Markovic, C.D. Friedman, P.D. Costantino, J. Biomed. Mater. Res., 58, 36 (2001).

13. J.E. Barralet, T. Gaunt, A.J. Wright, I.R. Gibson, J.C. Knowles, J. Biomed. Mater. Res., 63, 1 (2002).

14. L.L. Hench, J. Wilson, Introduction. An introduction to bioceramics. Ed. by L.L. Hench, J. Wilson, 1 (1993). 